\title{
An analytical study of ambiguity decorrelation using dual frequency code and carrier phase
}

\author{
P. J. G. Teunissen \\ Delft Geodetic Computing Centre (LGR), Department of Geodetic Engineering, Delft University of Technology, \\ Thijsseweg 11, NL-2629 JA Delft, The Netherlands
}

Received 21 June 1995; Accepted 20 December 1995

\begin{abstract}
In this contribution the integer least-squares estimation of the double differenced $L_{1}$ and $L_{2}$ ambiguities is analyzed, under the provision that the relative receiver-satellite geometry is dispensed with. The variance-covariance matrix of the ambiguities is instrumental for gaining insight into the characteristics of the ambiguity fixing process. A qualitative geometric description in detail is therefore given of the ambiguity search space. The elongation, the correlation coefficient and the areas of the ambiguity search space and its enclosing boxes are all given as function of the ratio or product of the carrier phase and code standard deviations. It is shown that the ambiguity search space is very elongated and that the ambiguities are highly correlated. It is also shown how the high correlation between the ambiguities can be used to ones advantage for the transformation to new ambiguities. This is done by means of the least-squares ambiguity decorrelation adjustment method. The improvements in terms of decorrelation, elongation and precision are shown and the corresponding optimal time-invariant ambiguity transformations are given for a practical range of code and measurement precisions.
\end{abstract}

\section{Introduction}

High precision relative GPS positioning is based on the very precise carrier phase measurements. The GPS double difference carrier phase measurements are however ambiguous by an unknown integer number of cycles. This implies that the time span needed for precise positioning is determined to a large extent by the time span of data which is needed to resolve for the integer double difference ambiguities. A significant reduction in the required observational time span can be achieved, when the integer ambiguity estimation process is integrated with the leastsquares adjustment of the baseline. For most surveying applications, this is the common mode of operation. It allows for instantaneous or almost instanteneous positioning, depending on whether both code and carrier phase measurements or carrier phase measurements only are used, see e.g. [Blewitt, 1989], [Frei and Beutler, 1990], [Hatch, 1991], [Wübbena, 1991], [Euler and Landau, 1992], [Teunissen, 1993] and [Tiberius and de Jonge, 1995a]. The reduction in the required time span is achieved due to the inclusion into the integer estimation process of the relative receiver-satellite geometry. As a result, redundancy increases and therefore in general, a significant increase in likelihood of the integer least-squares solution is obtained.

Integer ambiguity estimation is however also possible when one opts for dispensing with the relative satellite-receiver geometry, see e.g. [Hatch, 1982], [Euler and Goad, 1990], [Dedes and Goad, 1994] and [Euler and Hatch, 1994]. In fact, from a conceptual point of view, this is the simplest approach to integer ambiguity estimation. The code measurements are then directly used to determine the unknown integer ambiguities of the observed carrier phases. In case the code measurements are of poor precision though, a major disadvantage of this technique when compared with the approach discussed above is the length of the observational time span needed to obtain sufficiently precise estimates of the carrier phase ambiguities. This is due to the fact that the variance of the estimated ambiguity is dominated by the variance of the code measurements divided by the number of measurement epochs used. Hence, reliable integer fixing of the ambiguity becomes feasible only when sufficiently precise code 
measurements are available and/or when a sufficient number of samples are taken.

Despite the mentioned drawback of directly using the code measurements for ambiguity fixing, it is felt that a systematic study of this rather straightforward technique is still warranted. The four major reasons for the author are: (a) the still ongoing technical developments for improving the precision of the code measurements; (b) to show how this technique fits into the general theoretical framework of ambiguity fixing; (c) to present results which are of an analytical nature; and (d) to allow for a comparison with the results that can be achieved when the relative receiversatellite geometry is incorporated into the ambiguity fixing process. In view of the increased relevance of code measurements, it becomes of importance to know to which extent reliable ambiguity fixing is feasible for different measurement scenarios. In order to gain a qualitative insight, use will be made of analytical results rather than only numerical results.

In section 2, both the single and dual frequency case will be considered. It will be assumed that the data pertain to relatively short baselines such that the ionospheric delays may be ignored. For the dual frequency case, the variancecovariance matrix of the ambiguities will be given. This matrix is instrumental for gaining a qualitative insight into the characteristics of computing the integer least-squares ambiguities. In section 3, the geometry of the ambiguity search space is analyzed. Diagnostic measures used, are: the correlation coefficient, the elongation and the areas of both the ambiguity search space and its enclosing boxes. These diagnostic measures have also been used by Teunissen [1994a] for characterizing the ambiguity search space in case the relative receiver-satellite is taken into account. Based on these diagnostic measures, the impact of the shape of the ambiguity search space on the integer least-squares estimation can be described. This is done in section 4 for the special case of exact carrier phase data and in section 5 for the general case. Finally in section 6 , the decorrelation of the $L_{1}$ and $L_{2}$ ambiguities is taken up by means of the least-squares ambiguity decorrelation adjustment. The Least-squares AMBiguity Decorrelation Adjustment (LAMBDA) has been introduced in [Teunissen, 1993]. It is a method for the efficient computation of the required integer ambiguities. The two main steps of the method are: (a) the decorrelation of the least-squares ambiguities; and (b) the sequential conditional least-squares based search. An elementary presentation of the basic principles of the method is given in [Teunissen et al., 1995] and [Tiberius and de Jonge, 1995b], and numerical results obtained with the method when applied to fast single baseline computations are given in e.g. [Teunissen, 1994a] and [Tiberius and de Jonge, 1995a]. In the present contribution, the decorrelation of the ambiguities is studied analytically as well as numerically. The class of ambiguity transformations which can be used for the decorrelation process, has been discussed in [Teunissen, 1995a] and an extensive review of the method is given in the lecture notes of the International School "GPS for Geodesy" [Teunissen, 1995b].

\section{The single and dual frequency case}

In order to provide for a reference, we will first consider the case where only single frequency data of code and carrier phases are available. If we assume these data to pertain to a relatively short baseline such that the ionospheric delays may be ignored, then - when expressed in units of range (rather than cycles) - the double difference carrier phase measurement on $L_{1}$ at time epoch $i, \Phi_{1}(i)$, and its corresponding double difference code measurement, $P_{1}(i)$, can be represented in their simplest form as

$$
\begin{aligned}
& \Phi_{1}(i)=\rho(i)+\lambda_{1} N_{1} \\
& P_{1}(i)=\rho(i)
\end{aligned}
$$

In this pair of equations, $\rho(i)$ includes all terms that are common to both $\Phi_{1}(i)$ and $P_{1}(i)$. Hence, $\rho(i)$ includes the double difference form of the range from receiver to satellite, as well as the tropospheric delay. The wavelength of the $L_{1}$ carrier is denoted as $\lambda_{1}\left(\lambda_{1} \cong 19 \mathrm{~cm}\right)$ and $N_{1}$ denotes the integer double difference carrier phase ambiguity of $\Phi_{1}$. The ambiguity $N_{1}$ is assumed to be constant in time.

The time-averaged real-valued estimate of the ambiguity follows from (1) as

$$
\hat{N}_{1}=\frac{1}{\lambda_{1} k} \sum_{i=1}^{k}\left(\Phi_{1}(i)-P_{1}(i)\right)
$$

The most likely integer estimate of the ambiguity is then obtained from a simple rounding of $\hat{N}_{1}$ to its nearest integer. In order to validate the most likely integer ambiguity estimate, we of course still need to infer the likelihood of the above integer estimate. Based on the assumptions that: (a) unmodelled effects in (1) are absent; (b) the variances of $\Phi_{1}(i)$ and $P_{1}(i)$ are time-invariant; and (c) $\Phi_{1}(i)$ and $P_{1}(i)$ are not cross correlated nor time correlated; an application of the error propagation law to 
(2), gives the variance of $\hat{N}_{1}$ as

$$
\sigma_{N_{1}}^{2}=\frac{4 \sigma_{P}^{2}}{\lambda_{1}^{2} k}\left(1+\sigma_{\Phi}^{2} / \sigma_{P}^{2}\right)
$$

Note that the variances $\sigma_{\Phi}^{2}$ and $\sigma_{P}^{2}$ in this expression, are the variances of the undifferenced carrier phase and code measurements. Since the precision of the code measurements is much poorer than that of the carrier phase measurements $\left(\sigma_{\Phi}<<\sigma_{P}\right)$, equation (3) clearly shows that the precision with which the ambiguity can be estimated, is dominated by the precision of the code measurements.

Based on a standard deviation of the undifferenced carrier phase measurement of $\sigma_{\Phi}=0.3 \mathrm{~cm}$, table 1 shows, for different values of $k$ and $\sigma_{p}$, some typical values the standard deviation of $\hat{N}_{1}$ can take. It will be clear from the results of this table, that a reliable integer fixing of the $L_{1}$ ambiguity becomes feasible only, when sufficiently precise code measurements are available and/or when a sufficient number of samples are taken. As an example consider a time spacing of 5 seconds between the samples. In case $\sigma_{P}$ $=30 \mathrm{~cm}$, this would then imply an 8.3 minutes observational time span, to achieve an ambiguity standard deviation of 0.3 cycles.

\begin{tabular}{l|cccc}
$\sigma_{\mathrm{N} 1}$ (cycle) & $\sigma_{\mathrm{p}}=60 \mathrm{~cm}$ & $\sigma_{\mathrm{P}}=45 \mathrm{~cm}$ & $\sigma_{\mathrm{P}}=30 \mathrm{~cm}$ & $\sigma_{\mathrm{P}}=10 \mathrm{~cm}$ \\
\hline$k=1$ & 6.3 & 4.7 & 3.2 & 1.1 \\
$k=10$ & 2.0 & 1.5 & 1.0 & 0.3 \\
$k=100$ & 0.6 & 0.5 & 0.3 & 0.1 \\
$k=1000$ & 0.2 & 0.1 & 0.1 & 0.03
\end{tabular}

Table 1: Standard deviations (in cycles) of the timeaveraged single frequency $L_{1}$ ambiguity estimates for varying $k$ and $\sigma_{P}$, with $\sigma_{\Phi}=0.3 \mathrm{~cm}$.

One can expect that the situation improves, in case dual frequency data are used. In the dual frequency case, the double difference equations for phase and code read

$$
\begin{aligned}
& \Phi_{1}(i)=\rho(i)+\lambda_{1} N_{1} \\
& \Phi_{2}(i)=\rho(i)+\lambda_{2} N_{2} \\
& P_{1}(i)=\rho(i) \\
& P_{2}(i)=\rho(i)
\end{aligned}
$$

These are four equations in three unknowns, with the redundancy of one being due to the presence of the code measurement on the second frequency. Again we will assume that the observables have time-invariant variances and that they are not cross correlated nor time-correlated. We will also assume that the variances of the observables are frequency independent. Thus, $\sigma_{\Phi_{1}}=\sigma_{\Phi_{2}}=\sigma_{\Phi}$ and
$\sigma_{P_{1}}=\sigma_{P_{2}}=\sigma_{P}$. The least-squares solution for the two ambiguities, based on a $k$ number of samples, follows then as

$$
\begin{aligned}
& \hat{N}_{1}=\frac{1}{\lambda_{1} k} \sum_{i=1}^{k}\left(\Phi_{1}(i)-\left(P_{1}(i)+P_{2}(i)\right) / 2\right) \\
& \hat{N}_{2}=\frac{1}{\lambda_{2} k} \sum_{i=1}^{k}\left(\Phi_{2}(i)-\left(P_{1}(i)+P_{2}(i)\right) / 2\right)
\end{aligned}
$$

Note that the two ambiguities are correlated due to the presence of the code measurements. The ambiguity variance-covariance matrix follows from an application of the error propagation law to (5) as

$$
\begin{aligned}
Q_{N} & =\left[\begin{array}{cc}
\sigma_{N_{1}}^{2} & \sigma_{N_{1} N_{2}} \\
\sigma_{N_{2} N_{1}} & \sigma_{N_{2}}^{2}
\end{array}\right] \\
& =\frac{2 \sigma_{P}^{2}}{\lambda_{1} \lambda_{2} k}\left[\begin{array}{cc}
\left(\lambda_{2} / \lambda_{1}\right)\left(1+2 \sigma_{\Phi}^{2} / \sigma_{P}^{2}\right) & 1 \\
1 & \left(\lambda_{1} / \lambda_{2}\right)\left(1+2 \sigma_{\Phi}^{2} / \sigma_{P}^{2}\right)
\end{array}\right)
\end{aligned}
$$

It is remarked that if one would replace $\sigma_{P}^{2}$ in (6) by $2 \sigma_{P}^{2}$, one would get the ambiguity variance-covariance matrix, that belongs to the model which uses dual frequency phase, but single frequency code. Note, if we neglect the carrier phase variance in (6), that, when compared to the single frequency case (3), the variances of the two ambiguities have improved by a factor of two. For a single epoch $(k=1)$, the standard deviations of the two ambiguities read in cycles: $\sigma_{N_{1}} \cong 4.46$ and $\sigma_{N_{2}} \cong 5.72$, when $\sigma_{\Phi}=0.3 \mathrm{~cm}$ and $\sigma_{P}=60 \mathrm{~cm}$, and $\sigma_{N_{1}} \cong 0.74$ and $\sigma_{N_{2}} \cong 0.95$, when $\sigma_{\Phi}$ $=0.3 \mathrm{~cm}$ and $\sigma_{P}=10 \mathrm{~cm}$. It should be stressed however, that in the present case, it is not sufficient to consider only the precision of the individual ambiguities. That is, the most likely integer estimates of the two ambiguities are not obtained from a simple rounding of the real-valued estimates of (5) to their nearest integer. Hence, in the dual frequency case it generally does not make much sense to come up with a table, like table 1, showing the standard deviations of the $L_{1}$ and $L_{2}$ ambiguities. This is due to the fact that the two ambiguities are correlated. Hence, in order to be able to come up with the most likely integer ambiguity estimates, the complete ambiguity variancecovariance matrix of the two ambiguities needs to be taken into account.

It is interesting to observe what can be said when, instead of (5), one would use the following ambiguity estimates 


$$
\begin{aligned}
& \hat{N}_{1}^{\prime}=\frac{1}{\lambda_{1} k} \sum_{i=1}^{k}\left(\Phi_{1}(i)-P_{1}(i)\right) \\
& \hat{N}_{2}^{\prime}=\frac{1}{\lambda_{2} k} \sum_{i=1}^{k}\left(\Phi_{2}(i)-P_{2}(i)\right)
\end{aligned}
$$

Note, that these two ambiguities are now uncorrelated. Hence, their ambiguity variance covariance matrix is diagonal, which implies that the two ambiguities can now be treated independently using the simple scheme of rounding to the nearest integer. This may seem to be an advantage. A closer look however, will reveal that this is not true. In order to see this, first note that $\hat{N}_{1}^{\prime}$ follows from a least-squares adjustment that is based on the first and third equation of (4) and that $\hat{N}_{2}^{\prime}$ follows from a leastsquares adjustment that is based on using the second and fourth equation of (4). But this implies, that one is not making use of a dual-frequency adjustment. Instead, one is applying a single frequency adjustment, which is executed twice for each of the two frequencies separately. As a result, one is disregarding the redundancy which is present in the four equations of (4). Disregarding redundancy implies a neglect of information, which otherwise could have been put to a good use in the ambiguity fixing process. The important point to understand is therefore, that the correlation present in (5) is to our advantage and not to our disadvantage. It is to our advantage in the sense that with (5) one will be more successful when validating the integer least-squares ambiguities, then when using (7). Of course with (5), one cannot use the simple scheme of rounding to the nearest integer. But this does not matter, since the transformation and search step of the LAMBDAmethod takes care of this situation in a very efficient manner. In fact, as it will be shown in the sections following, the ambiguity transformation of the LAMBDAmethod makes use of the existing non-zero correlation, so as to come up with new ambiguities that are largely decorrelated and that are also much more precise than the original ambiguities.

\section{The geometry of the ambiguity search space}

In order to get a better understanding of the relevance and implications of using the complete variance-covariance matrix, this section will introduce diagnostics for characterizing the size and shape of the ambiguity search space. The ambiguity search space is defined as the set of vectors $N \in R^{2}$ that satisfy the inequality

$$
(\hat{N}-N)^{*} Q_{N}^{-1}(\hat{N}-N) \leq \chi^{2}
$$

with $\hat{N}=\left(\hat{N}_{1}, \hat{N}_{2}\right)^{*}$ and $\chi^{2}$ being a suitably chosen constant. The ambiguity search space is ellipsoidal and centred at $\hat{N}$. The shape of the search space is governed by the variance-covariance matrix $Q_{N}$ and its size can be controlled by $\chi^{2}$. First we will give an indication of what one can expect from the shape of the ambiguity search space. After that, we will consider the elongation of the ambiguity search space, the ambiguity correlation coefficient and the areas of the ambiguity search space and its enclosing boxes.

\section{Shape of ambiguity search space}

A first indication of the shape of the ambiguity search space is obtained from the observation that the ambiguity variance-covariance matrix (6) can be written as the sum of two special matrices:

$$
\begin{aligned}
Q_{N} & =\frac{4 \sigma_{\Phi}^{2}}{\lambda_{1} \lambda_{2} k}\left[\begin{array}{cc}
\lambda_{2} / \lambda_{1} & 0 \\
0 & \lambda_{1} / \lambda_{2}
\end{array}\right] \\
& +\frac{2 \sigma_{P}^{2}}{\lambda_{1} \lambda_{2} k}\left[\begin{array}{l}
\left(\lambda_{2} / \lambda_{1}\right)^{1 / 2} \\
\left(\lambda_{1} / \lambda_{2}\right)^{1 / 2}
\end{array}\right]\left[\begin{array}{l}
\left(\lambda_{2} / \lambda_{1}\right)^{1 / 2} \\
\left(\lambda_{1} / \lambda_{2}\right)^{1 / 2}
\end{array}\right]^{*}
\end{aligned}
$$

The first matrix in this sum is diagonal, having entries which are very small due to the high precision of the carrier phase measurements. The second matrix in this sum is of rank 1 . Due to the relatively poor precision of the code measurements, its entries are large compared to the entries of the first matrix. This type of decomposition, where the variance-covariance matrix of the ambiguities can be written as the sum of a full rank matrix and a matrix of less than full rank, is very typical for GPS. The relevance of this type of decomposition in terms of the shape of the spectrum of conditional ambiguity variances was already stressed by the author in [Teunissen, 1993]. Its implications for the computation of the integer least-squares ambiguities are shown in [Teunissen, 1994b] and [Teunissen et al., 1994]. Analytically worked out synthetic examples are given in [Teunissen, 1995b].

The variance-covariance matrix $Q_{N}$ is diagonal when $\sigma_{P}=$ 0 . Hence, in that case, correlation between the ambiguities will be absent and the principal axes of the ambiguity search space will be aligned with the grid axes. In practice however, this will not be the case since $\sigma_{P}>0$. In fact, since $\sigma_{\Phi} \ll \sigma_{P}$ in practice, a direct consequence of the above decomposition, is that the ambiguity search space must be very outstretched. That is, it will be very narrow in one direction and rather lengthy in the other orthogonal 
direction. This can be seen as follows. Assume for the moment that $\sigma_{\Phi}=0$. The matrix $Q_{N}$ will then fail to be of full rank. In fact it is of rank 1. This implies that the corresponding ambiguity search space, instead of being ellipsoidal, collapses to a single straight line interval. Now assume that $\sigma_{\Phi}$ is positive, but still very small. The matrix $Q_{N}$ will then theoretically be of full rank. Numerically however, it will have a near rankdefect. The vector $e_{1}=\left(\left(\lambda_{1} / \lambda_{2}\right)^{1 / 2},-\left(\lambda_{2} / \lambda_{1}\right)^{1 / 2}\right)^{*}$, which is orthogonal to the column vector of the above rank-1 matrix, may then be considered an approximation to the eigenvector of $Q_{N}$ belonging to the smallest eigenvalue. Using $e_{1}{ }^{*} Q_{N} e_{1} / e_{1}{ }^{*} e_{1}=\mu_{1}$, the smallest eigenvalue can be approximated as

$$
\mu_{1}=8 \frac{\sigma_{\Phi}^{2}}{k}\left(1 /\left(\lambda_{1}^{2}+\lambda_{2}^{2}\right)\right)
$$

Since $\mu_{1}$ is very small indeed, it follows that the ambiguity search space will be very narrow in the direction of $e_{1}$. The column vector of the above rank-1 matrix itself, $e_{2}=\left(\left(\lambda_{2} / \lambda_{1}\right)^{1 / 2},\left(\lambda_{1} / \lambda_{2}\right)^{1 / 2}\right)^{*}$ may be considered to approximate the eigenvector of $Q_{N}$ belonging to the largest eigenvalue. Using $e_{2}^{*} Q_{N} e_{2} / e_{2}^{*} e_{2}=\mu_{2}$ and neglecting $\sigma_{\Phi}$, the largest eigenvalue can be approximated as

$$
\mu_{2}=2 \frac{\sigma_{P}^{2}}{k}\left(1 / \lambda_{1}^{2}+1 / \lambda_{2}^{2}\right)
$$

From (10) and (11) follows that the small eigenvalue is governed by the precision of phase, whereas the large eigenvalue is governed by the precision of code. Since $\mu_{2}>\mu_{1}$, it follows that the ambiguity search space can indeed be considered to be outstretched in the $e_{2}$-direction.

\section{Elongation of the ambiguity search space}

The elongation $e_{N_{1} N_{2}}$ is a numerical measure for the shape of the ambiguity search space. The elongation is defined as the ratio of the length of the major axis and the length of the minor axis of the ambiguity search space. Hence, it equals the square-root of the ratio of the largest and smallest eigenvalue of $Q_{N}$. The elongation follows from (6) as

$$
e_{N_{1} N_{2}}=\left(\frac{1+\left(1-r^{2}\right)^{1 / 2}}{1-\left(1-r^{2}\right)^{1 / 2}}\right)^{1 / 2}
$$

with

$$
r=4\left(\left(1+\sigma_{P}^{2} / \sigma_{\Phi}^{2}\right)^{-1 / 2}+\left(1+\sigma_{P}^{2} / \sigma_{\Phi}^{2}\right)^{1 / 2}\right)^{-1}\left(\lambda_{1} / \lambda_{2}+\lambda_{2} / \lambda_{1}\right)^{-1} .
$$

Note that the elongation is independent of $k$. Hence, the number of samples used will not effect the shape of the ambiguity search space. Also note that the elongation is uniquely determined by the variance ratio $\sigma_{\Phi}^{2} / \sigma_{p}^{2}$. In general, elongation lies in the interval $[1, \infty)$. The ellipse reduces to a circle when $e=1$ and it collapses to a straight line when $e \rightarrow \infty$. In our case, we have $e_{N_{1} N_{2}}=1$ when $r=1$ and $e_{N_{T_{2}}}=\infty$ when $r=0$. The case $r=0$ corresponds with $\sigma_{P} / \sigma_{\Phi}=\infty$. This shows that the ambiguity search space will be very elongated when the precision of the code is poor with respect to the precision of phase or, vice versa, when the precision of phase is very high with respect to the precision of code. Since in practice we indeed have $\sigma_{\Phi}<<\sigma_{P}$, one can expect that the actual ambiguity search space is very elongated. For $\sigma_{\Phi}=0.3 \mathrm{~cm}$ and $\sigma_{P}=60 \mathrm{~cm}$, we have $e_{N_{,} N_{2}} \cong 206$. This shows that if the minor axis of the search space would be $1 \mathrm{~cm}$ long, its major axis would be about 2 meters long. The elongation is pushed towards its minimum value of one, the smaller the ratio $\sigma_{p} / \sigma_{\Phi}$ gets. Hence, the ambiguity search space will become less elongated the more precise the code measurements are, relative to the precision of the carrier phase measurements. In our case however, the minimum value $e_{N_{1} N_{2}}=1 \mathrm{can}$ never be reached. The closest we can get to this value is when $\sigma_{P} / \sigma_{\Phi}=0$. In that case $r$ reaches its maximum value of $r=2 /\left(\lambda_{1} / \lambda_{2}+\lambda_{2} / \lambda_{1}\right)$ and the elongation reaches its minimum value of $e_{N_{1} N_{2}}=\lambda_{2} / \lambda_{1}$.

\section{The ambiguity correlation coefficient}

As a measure for the dependency between $\hat{N}_{1}$ and $\hat{N}_{2}$, we consider the correlation coefficient $\rho_{N_{i} N_{2}}$. As the elongation, the correlation coefficient is dimensionless. It follows from (6) as

$$
\rho_{\hat{N}_{1} \hat{N}_{2}}=\left(1+2 \sigma_{\Phi}^{2} / \sigma_{P}^{2}\right)^{-1}
$$

This result shows, as it was the case with the elongation, that the correlation coefficient is independent of $k$ and that it is uniquely determined by the variance ratio $\sigma_{\Phi}^{2} / \sigma_{P}^{2}$. The correlation coefficient is thus determined by the precision of the phase measurements relative to the precision of the code measurements. A change in code variance from $(60 \mathrm{~cm})^{2}$ to $(10 \mathrm{~cm})^{2}$ is a major improvement for the precision of the code measurements, but it will increase the variance ratio $\sigma_{\Phi}^{2} / \sigma_{P}^{2}$ by the same factor - 36 in this case as when the carrier phase variance changes from $(1 \mathrm{~mm})^{2}$ to $(6 \mathrm{~mm})^{2}$.

It follows from (13), since $\sigma_{\Phi}<<\sigma_{P}$ in practice, that the two ambiguities $\hat{N}_{1}$ and $\hat{N}_{2}$ will be highly correlated indeed. In fact, when $\sigma_{\Phi}=0.3 \mathrm{~cm}$, we have $\rho_{\hat{N_{1} \hat{N}_{2}}}=0.99995$ for $\sigma_{P}=60 \mathrm{~cm}$ and $\rho_{N_{1} N_{2}}=0.9982$ for $\sigma_{P}=10 \mathrm{~cm}$, showing that the correlation coefficient stays very close to 1 for the code precision range considered. Since $\left(1-\rho_{N_{1} N_{2}}^{2}\right)=$ $\sigma_{\hat{N}_{2} \mid \hat{N}_{1}}^{2} / \sigma_{\hat{N}_{2}}^{2}=\sigma_{\hat{N}_{1} \mid \hat{N}_{2}}^{2} / \sigma_{\hat{N}_{1}}^{2}$, with $\sigma_{\hat{N}_{2} \mid N_{1}}^{2}$ and $\sigma_{\hat{N}_{1} \mid N_{2}}^{2}$ being the 
conditional variances, the high correlation implies that the conditional variances of the two ambiguities are very much smaller than their unconditional counterparts. The conditional variance of the $L_{2}$ ambiguity reads for instance

$$
\sigma_{\hat{N}_{2} \mid \hat{N}_{1}}^{2}=\frac{8 \sigma_{\Phi}^{2}}{\lambda_{2}^{2} k}\left(1+\left(1+\sigma_{P}^{2} / \sigma_{\Phi}^{2}\right)^{-1}\right)^{-1} .
$$

From this it follows for $k=1$, that $\sigma_{\hat{N}_{\mid} \mid \hat{N}_{1}} \cong 0.03$ cycle, when $\sigma_{\Phi}=0.3 \mathrm{~cm}$ and $\sigma_{P}=60 \mathrm{~cm}$. This shows, that once the first ambiguity is known, the second ambiguity can be fixed to an integer with a high confidence.

\section{Area of search space, eigenvalue-box and sigma-box}

In [Teunissen, 1993], the author introduced the area of the ambiguity search space as a measure to indicate the number of grid points located in the search space. As such it can be used as a reliability measure for validation. For instance, when the area of the ambiguity search space is small enough, such that only one single grid point is located in it, one can expect validation to be more successful. Figure la shows the ellipsoidal ambiguity search space and its best fitting 2-dimensional box. Since the two sides of this box are determined by the two eigenvalues of the variancecovariance matrix $Q_{N}$, this box will be referred to as the eigenvalue-box. The area of the ambiguity search space, $A_{N}$, and the area of the eigenvalue-box, $A_{\mu_{N}}$, are both determined by the determinant of $Q_{N}$. They read

$$
A_{N}=\pi \chi^{2}\left(\operatorname{det} Q_{N}\right)^{1 / 2} \text { and } A_{\mu_{N}}=4 \chi^{2}\left(\operatorname{det} Q_{N}\right)^{1 / 2}
$$

The determinant of $Q_{N}$ follows from (6) as

$$
\operatorname{det}\left(Q_{N}\right)=\frac{16 \sigma_{\Phi}^{2} \sigma_{P}^{2}}{\lambda_{1}^{2} \lambda_{2}^{2} k^{2}}\left(1+\sigma_{\Phi}^{2} / \sigma_{P}^{2}\right) \text {. }
$$

The determinant does, of course, depend on $k$. The more samples are used, the smaller the ambiguity search space becomes. For $k=1, \sigma_{\Phi}=0.3 \mathrm{~cm}$ and $\sigma_{P}=60 \mathrm{~cm}$, we have $\left(\operatorname{det} Q_{N}\right)^{1 / 2}=0.155$, from which it follows that $A_{N}=4.87$ (cycles) $^{2}$ and $A_{\mu_{N}}=6.21$ (cycles) $^{2}$ for $\chi^{2}=10$. The value $A_{N}=4.87$ (cycles) $^{2}$ indicates, that the number of grid points inside the ambiguity search space can be expected to be of the order of five. When $A_{N}$ is significantly smaller than one, one can expect that the ambiguity search space contains only one grid point. Table 2 gives the range of values the area $A_{N}$ can typically take. Assuming that the data are normally distributed, the value $\chi^{2}=10$ corresponds, in the two dimensional case with a level of significance slightly smaller than 0.01 , whereas the value $\chi^{2}$ $=15$ corresponds with a level of significance slightly larger than 0.0005 .

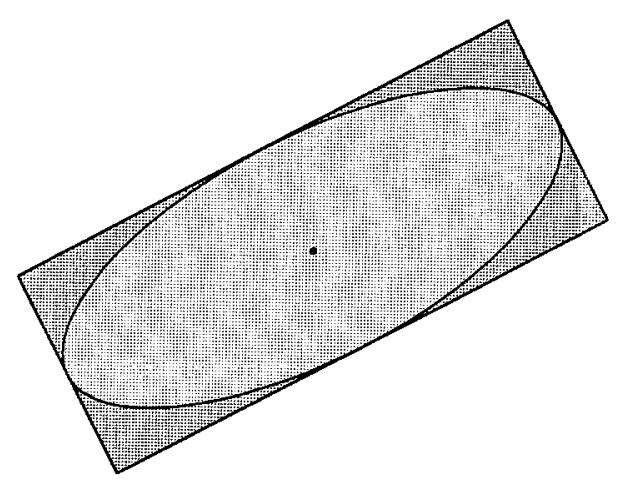

(a) Ambiguity search space with area $A_{N}$ and eigenvalue-box with area $A_{\mu_{N}}$

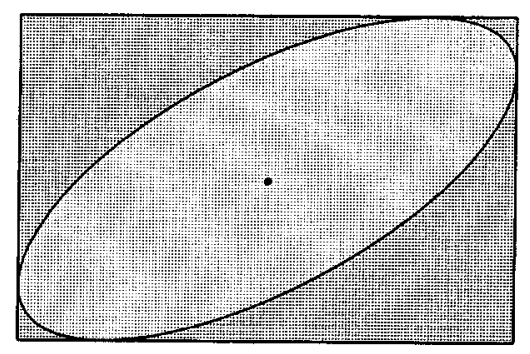

(b) Ambiguity search space with area $A_{N}$ and sigma-box with area $A_{\sigma}$

Figure 1: The areas of the ambiguity search space, eigenvalue-box and sigma-box.

\begin{tabular}{|c|cc|cc|}
\hline$A_{N}$ & \multicolumn{2}{|c|}{$\chi^{2}=10$} & \multicolumn{2}{c|}{$\chi^{2}=15$} \\
$\left(\sigma_{\Phi}=0.3 \mathrm{~cm}\right)$ & $\sigma_{p}=60 \mathrm{~cm}$ & $\sigma_{P}=10 \mathrm{~cm}$ & $\sigma_{P}=60 \mathrm{~cm}$ & $\sigma_{P}=10 \mathrm{~cm}$ \\
\hline$k=1$ & 4.87 & 0.81 & 7.30 & 1.22 \\
$k=2$ & 2.43 & 0.41 & 3.64 & 0.61 \\
$k=10$ & 0.49 & 0.08 & 0.73 & 0.12 \\
\hline
\end{tabular}

Table 2: The area of the ambiguity search space, $A_{N}$.

The 2-dimensional box that encloses the ambiguity search space and which has its sides parallel to the grid axes, will be referred to as the sigma-box. Its two sides are determined by the standard deviations of the two ambiguities. Figure $1 \mathrm{~b}$ shows the ellipsoidal ambiguity search space and the sigma-box. The area of the sigma-box is given as

$$
A_{\sigma_{N}}=4 \chi^{2} \sigma_{N_{1}} \sigma_{N_{2}}
$$

It will be clear that the sigma-box and eigenvalue-box become identical only when the principal axes of the ambiguity search space are aligned with the grid axes. The ratio of the two areas $A_{\mu_{N}}$ and $A_{\sigma_{N}}$ can therefore be used as a measure for the way the sigma-box fits the ambiguity search space. A best fit would then correspond to a value 
of one for this ratio and a poor fit with a value being close to zero. Using (15) and (17) it follows that

$$
\frac{A_{\mu_{N}}}{A_{\sigma_{N}}}=\frac{4 \chi^{2}\left(\operatorname{det} Q_{N}\right)^{1 / 2}}{4 \chi^{2} \sigma_{N_{1}} \sigma_{N_{2}}}=\left(1-\rho_{N_{1} N_{2}}^{2}\right)^{1 / 2} .
$$

This result shows that the ratio of $A_{\mu_{r}}$ and $A_{\sigma_{N}}$ is uniquely determined by the ambiguity correlation coefficient. Hence, besides the usual statistical interpretation, also an interesting geometrical interpretation can be given to the correlation coefficient. Since we have shown already, that in our case the correlation coefficient will be close to one, it follows from (18) that the area of the sigma-box will by far exceed the area of the eigenvalue-box $\left(A_{\sigma_{N}}>>A_{\mu_{N}}\right)$. In fact, for $\sigma_{\Phi}=0.3 \mathrm{~cm}$ and $\sigma_{P}=60 \mathrm{~cm}$, the area of the sigma-box will be about one hundred times larger than the area of the eigenvalue-box. Hence, in our case the sigmabox indeed fits the ambiguity search space rather poorly.

In this section, the characteristics of the ambiguity search space have been studied by means of the elongation, the correlation coefficient and the area of the ambiguity search space. We will now combine these three measures and show how they relate to the precision of the ambiguities. This will be done for the arithmetic and geometric mean of the ambiguity variances, respectively $\left(\sigma_{N_{1}}^{2}+\sigma_{N_{2}}^{2}\right) / 2$ and $\left(\sigma_{N_{1}}^{2} \sigma_{N_{2}}^{2}\right)^{1 / 2}$. The arithmetic and geometric mean are determined by the elongation $\left(e_{N_{1} N_{2}}\right)$, the correlation coefficient $\left(\rho_{N_{N} N_{2}}\right)$ and the area of the ambiguity search space $\left(A_{N}\right)$ as

$$
\begin{aligned}
& \frac{1}{2}\left(\sigma_{N_{1}}^{2}+\sigma_{N_{2}}^{2}\right)=\frac{A_{N}}{2 \pi \chi^{2}}\left(e_{N_{1} N_{2}}+e_{N_{1} N_{2}}^{-1}\right) \\
& \left(\sigma_{N_{1}}^{2} \sigma_{N_{2}}^{2}\right)^{1 / 2}=\frac{A_{N}}{\pi \chi^{2}}\left(1-\rho_{N_{1} N_{2}}^{2}\right)^{-1 / 2}
\end{aligned}
$$

This result clearly shows how the precision of the ambiguities can be improved, by reducing the elongation and correlation coefficient. In the next and following sections, we will consider the construction of ambiguity transformations that improve the elongation and correlation, but that leave the area $A_{N}$ invariant.

\section{The 2D-decorrelating ambiguity transformation}

As it was pointed out above, a simple rounding of the realvalued estimates of the two ambiguities to their nearest integer values will not guarantee that the required most likely integer estimates of these two ambiguities is obtained. This will only be the case, when the ambiguities are completely decorrelated. Since the two ambiguities are correlated in our case, the complete variance-covariance matrix of the ambiguities needs to be taken into account when computing the most likely integer estimates. Following [Teunissen, 1993], the computation of the most likely integer ambiguity estimates makes use of a search which is based on bounds that follow from a sequential conditional least-squares adjustment of the ambiguities. When expressed in terms of the $L_{1}$ and $L_{2}$ ambiguities, these two bounds read as

$$
\left\{\begin{array}{l}
\left(\hat{N}_{1}-N_{1}\right)^{2} \leq \sigma_{N_{1}}^{2} \chi^{2} \\
\left(\hat{N}_{2 \mid 1}-N_{2}\right)^{2} \leq \sigma_{N_{2} \mid N_{1}}^{2} \lambda\left(N_{1}\right) \chi^{2}
\end{array}\right.
$$

in which $\hat{N}_{2 \mid 1}=\hat{N}_{2}-\sigma_{N_{2} N_{1}} \sigma_{N_{1}}^{-2}\left(\hat{N}_{1}-N_{1}\right)$ and $\lambda\left(N_{1}\right)=1-$ $\left(\hat{N}_{1}-N_{1}\right)^{2} / \sigma_{N_{1}}^{2} \chi^{2}$. The search for the most likely integer ambiguity estimates, can briefly be described as follows (for more details, see e.g. [Teunissen, 1993, 1994b]). First one selects an integer ambiguity $N_{1}$ that satisfies the first bound of (20). Then, based on this chosen integer value $N_{1}$, the conditional least-squares estimate $\hat{N}_{2 \mid 1}$ and scalar $\lambda\left(N_{1}\right)$ are computed. These values are then used to select an integer ambiguity $\hat{N}_{2}$ that satisfies the second bound of (20). A restart is used when one fails to find an integer $N_{2}$ that satisfies this second bound. This is continued until an admissible integer-pair $\left(N_{1}, N_{2}\right)$ is found. Then a shrinking of the ambiguity search space can be applied through an appropriate downscaling of $\chi^{2}$, after which one can continue with the search process. This process is continued until one fails to find any remaining admissible integerpairs. The last found integer-pair is then the sought for most likely integer solution, being the integer least-squares estimate.

From (6) it follows that

$$
\sigma_{N_{2} \mid N_{1}}^{2} / \sigma_{N_{1}}^{2}=\frac{\lambda_{1}^{2}}{\lambda_{2}^{2}}\left(1-\rho_{N_{1} N_{2}}^{2}\right),
$$

which shows, since $\rho_{N_{1} N_{2}}$ is close to one, that $\sigma_{N_{2} \mid N_{1}}^{2}<<\sigma_{N_{1}}^{2}$. For instance, for $k=1, \sigma_{\Phi}=0.3 \mathrm{~cm}$ and $\sigma_{P}=60 \mathrm{~cm}$, we have that $\sigma_{N_{1}} \cong 4.5$ cycle and $\sigma_{N_{2} \mid N_{1}} \cong 0.03$ cycle. With reference to the bounds of (20), this implies that the potential of search halting is significant when one goes from the first to the second bound. As a consequence, a number of trials in the search are required, before one is able to find the integer least-squares solution. This situation can however be improved if we make use of the decorrelating ambiguity transformation $Z^{*}$ of the LAMBDA-method. This transformation transforms the original integer ambiguity vector $N=\left(N_{1}, N_{2}\right)^{*}$, its real- 
valued least-squares estimate $\hat{N}=\left(\hat{N}_{1}, \hat{N}_{2}\right)^{*}$ and its variance-covariance matrix $Q_{N}$ as

$$
z=Z^{*} N, \hat{z}=Z^{*} \hat{N}, Q_{z}=Z^{*} Q_{N} Z .
$$

The letter $z$ is used to denote the transformed ambiguities. The ambiguity transformation $Z^{*}$ has integer entries and is area preserving. The area preserving property implies that $Z^{*}$ leaves the areas of both the ambiguity search space and of its best fitting box invariant. The ambiguity transformation $Z^{*}$ is constructed from a sequence of Gaussian transformations of the following two types:

$$
Z_{1}^{*}=\left[\begin{array}{cc}
1 & 0 \\
z_{21} & 1
\end{array}\right) \text { and } Z_{2}^{*}=\left(\begin{array}{cc}
1 & z_{12} \\
0 & 1
\end{array}\right) \text {, }
$$

in which $z_{21}$ and $z_{12}$ are appropriately chosen integers, see [Teunissen, 1993, 1994a]. These integers are chosen such that the transformed ambiguities become less correlated and more precise. They are taken as the nearest integer to the ratio of the covariance and variance, times minus one. As a result of the ambiguity transformation, the original ambiguity search space is transformed into a new, but more sphere-like, ambiguity search space.

In order to show that the transformed ambiguity search space is more sphere-like than the original one, consider expression (12) with $r^{2}=4 \operatorname{det}\left(Q_{N}\right) /\left(\sigma_{N_{i}}^{2}+\sigma_{N_{2}}^{2}\right)^{2}$. Since the decorrelating ambiguity transformation is area preserving and improves the precision of the ambiguities, the transformation $Z^{*}$ increases the value of $r^{2}$. As a result the elongation $e_{N_{1} N_{2}}$ gets smaller.

In [Teunissen, 1993, 1994a] it was shown that the absolute value of the correlation coefficient of the transformed ambiguities is always less than or equal to one half. This implies that one can expect the ambiguity transformation to decorrelate the ambiguities if the correlation coefficient of the original ambiguities is larger than one half in absolute value. It follows from (13) that $\rho_{\hat{N}_{1} \hat{N}_{2}} \geq 1 / 2$, whenever $\sigma_{\Phi}^{2} / \sigma_{P}^{2} \leq 1 / 2$. This condition on the variance ratio is easily met in practice. Hence, one can indeed expect that our ambiguity transformation $Z^{*}$ will be able to decorrelate the $L_{1}$ and $L_{2}$ ambiguities.

\section{Ambiguity decorrelation in case of exact phase data}

Before considering the general case of ambiguity decorrelation, we will first consider the special case that $\sigma_{\Phi}=0$. We know that the carrier phase measurements are of a very high precision. The standard deviation of the undifferenced carrier phase measurements is only of the order of a few millimeters. From a theoretical point of view it is therefore of interest to consider the limiting case $\sigma_{\Phi}=0$. Once the results are obtained for this limiting case, one may consider whether they are also applicable for the practical range the code and carrier phase standard deviations take.

In (9) the variance-covariance matrix of the ambiguities, $Q_{N}$, has been decomposed into a sum of a diagonal full rank matrix and a rank-1 matrix. If $\sigma_{P}=0$, then $Q_{N}$ reduces to a diagonal matrix and both ambiguities become fully decorrelated. Hence, in this limiting case the decorrelating ambiguity transformation is simply the trivial identity. If $\sigma_{\Phi}=0$, then $Q_{N}$ reduces to a rank-1 matrix and the ambiguity search space collapses to a line interval. In this case the two ambiguities are fully correlated. If we write $Q_{N}$ as $Q_{N}=R_{2}+R_{1}$, with $R_{2}$ being the diagonal rank-2 matrix and $R_{1}$ being the rank-1 matrix, it follows from (9), using $\lambda_{2} / \lambda_{1}=77 / 60$, that

$$
R_{\mathrm{I}}=\frac{2}{(60)^{2}} \frac{\sigma_{P}^{2}}{\lambda_{2}^{2} k}\left(\begin{array}{l}
77 \\
60
\end{array}\right)\left(\begin{array}{l}
77 \\
60
\end{array}\right)^{*} .
$$

Hence, this would be the ambiguity variance-covariance matrix in case $\sigma_{\Phi}=0$. It would then also follow that

$$
-60 \hat{N}_{1}+77 \hat{N}_{2}=\text { constant } .
$$

This relation may now be used to directly construct the decorrelating ambiguity transformation $Z^{*}$. Hence, in this special case we do not need to construct $Z^{*}$ on the basis of a sequence of Gaussian transformations. It follows from (24) that the first row of $Z^{*}$ is given by $(-60,77)$. To obtain the second row of $Z^{*}$, we make use of the fact that all entries of $Z^{*}$ need to be integer and that $Z^{*}$ needs to be area preserving. This gives as second row of $Z^{*},(-7$, 9). The decorrelating transformation reads therefore as

$$
Z^{*}=\left[\begin{array}{rr}
-60 & 77 \\
-7 & 9
\end{array}\right] \text {. }
$$

Instead of $(-7,9)$, one may also take $(-7-i 60,9+i 77), i$ $=0, \pm 1, \pm 2 \ldots$. We have chosen $(-7,9)$ however, since it will give the best precision for the second ambiguity. If we apply the error propagation law, it follows from (23) and (25) that 


$$
Z^{*} R_{1} Z=\frac{2}{(60)^{2}} \frac{\sigma_{p}^{2}}{\lambda_{2}^{2} k}\left(\begin{array}{ll}
0 & 0 \\
0 & 1
\end{array}\right)
$$

This would then be the variance-covariance matrix of the transformed ambiguities, in case $\sigma_{\Phi}=0$. If we compare (23) with (26), we observe that

$$
\left\{\begin{array}{rll}
\rho_{N_{1} N_{2}}=1 & \Rightarrow & \rho_{z_{1} z_{2}}=0 \\
\sigma_{N_{1}}^{2}=2 \sigma_{P}^{2} / \lambda_{1}^{2} k & \Rightarrow & \sigma_{z_{1}}^{2}=0 \\
\sigma_{N_{2}}^{2}=2 \sigma_{P}^{2} / \lambda_{2}^{2} k & \Rightarrow & \sigma_{z_{2}}^{2}=\sigma_{N_{2}}^{2} /(60)^{2}
\end{array}\right.
$$

Hence, the ambiguity transformation $Z^{*}$ of (25) achieves a perfect decorrelation and a drastic improvement in precision. Integer estimation and validation of the transformed ambiguities becomes now rather straightforward. Due to the perfect decorrelation, the integer least-squares estimates of the two new ambiguities simply follow from rounding their real-valued estimates to their nearest integer. Also, validation would not be problematic, since $\sigma_{z_{1}}=0$ and $\sigma_{z_{2}}$ is as small as 0.06 cycle in case $\sigma_{p}=60 \mathrm{~cm}$ and $k=1$.

The above results are strictly valid of course, only for the case $\sigma_{\Phi}=0$. It is therefore of interest to infer whether the ambiguity transformation (25) performs for the general case as well as it does for the limiting case $\sigma_{\Phi}=0$. Thus instead of $Z^{*} R_{1} Z$, we now consider $Z^{*} Q_{N} Z=Z^{*}\left(R_{2}+\right.$ $\left.R_{1}\right) Z$, with $\sigma_{\Phi} \neq 0$. It follows from (9) and (25) that

$$
Z^{*} Q_{N} Z=\frac{2}{\lambda_{1} \lambda_{2} k}\left(\begin{array}{cc}
2 \sigma_{\Phi}^{2}(9240) & 2 \sigma_{\Phi}^{2}(1079) \\
2 \sigma_{\Phi}^{2}(1079) & \left(2 \sigma_{\Phi}^{2}(582121)+\sigma_{P}^{2}\right) / 4620
\end{array}\right]
$$

A comparison of this transformed variance-covariance matrix with the original variance-covariance matrix $Q_{N}$, shows for $k=1, \sigma_{\Phi}=0.3 \mathrm{~cm}$ and $\sigma_{P}=60 \mathrm{~cm}$, that

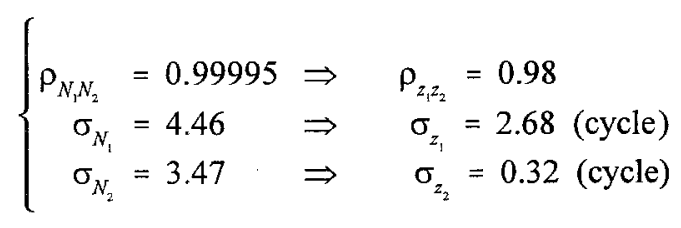

This shows that transformation (25) does improve the situation, but unfortunately however, the improvement is only marginal. In particular the correlation coefficient of the two transformed ambiguities is still very close to one. Hence, further improvements are possible, since we know that a correlation coefficient of less than one half in absolute value can be achieved. The conclusion reads therefore, that although $\sigma_{\Phi}=0.3 \mathrm{~cm}$ is small with respect to $\sigma_{P}=60 \mathrm{~cm}$, it is not small enough for the limiting case $\sigma_{\Phi}$ $=0$ to be valid. It follows from (28) that

$$
\rho_{z_{1} z_{2}}<1 / 2 \Leftrightarrow \sigma_{p}>1869 \sigma_{\Phi} .
$$

This shows that either the code precision must be rather poor or the carrier phase precision very high (e.g. $\sigma_{\Phi}=$ $0.1 \mathrm{~cm}, \sigma_{F}=187 \mathrm{~cm}$ ), in order to have a correlation coefficient of less than one half. In case of GPS however, neither the code precision is that poor, nor the carrier phase precision is that high.

\section{6. $L_{1} / L_{2}$ ambiguity decorrelation: the general case}

In this section we will consider the general case of decorrelating the $L_{1} / L_{2}$ ambiguities. Use will be made of the least-squares ambiguity decorrelation adjustment, as discussed in section 4 . But, before actually constructing the decorrelating ambiguity transformation, it is of interest to infer first the improvement that one can expect from the transformation. We know that the absolute value of the correlation coefficient of the transformed ambiguities is always less than or equal to one half. This implies, when we make use of relation (18) for the transformed ambiguity search space, that

$$
A_{\sigma_{z}} \leq \frac{2}{\sqrt{3}} A_{\mu_{z}} .
$$

Hence, instead of the factor of about one hundred between $A_{\sigma_{v}}$ and $A_{\mu_{v}}$, which we had earlier, the area of the sigmabox of the transformed ambiguity search space is now far closer to the area of the best fitting box. Based on this result, we can now also determine a bound on the ratio of $A_{\sigma_{\varepsilon}}$ and $A_{\sigma_{N}}$. Since the ambiguity transformation preserves the area of the best fitting box, $A_{\mu}=A_{\mu_{\mathrm{v}}}$, it follows from (6), (18) and (31) that $A_{\sigma_{z}} \leq(2 / \sqrt{3})\left(\operatorname{det} Q_{N}\right)^{1 / 2} A_{\sigma_{N}} /\left(\sigma_{N_{1}} \sigma_{N_{2}}\right)$ or that

$$
A_{\sigma_{z}} \leq \frac{4}{\sqrt{3}} A_{\sigma_{N}}\left(\left(1+\sigma_{P}^{2} / \sigma_{\Phi}^{2}\right)^{-1 / 2}+\left(1+\sigma_{P}^{2} / \sigma_{\Phi}^{2}\right)^{1 / 2}\right)^{-1} .
$$

This shows that the improvement is particularly pronounced when the variance ratio $\sigma_{P}^{2} / \sigma_{\Phi}^{2}$ is large. For $\sigma_{\Phi}=0.3 \mathrm{~cm}$ and $\sigma_{P}=60 \mathrm{~cm}$, we have $A_{\sigma_{s}} \leq 0.01 A_{\sigma_{N}}$. Assuming that the variances of the two transformed ambiguities are of the same order, this result shows that one can expect an improvement by a factor of ten for the ambiguity standard 
deviations.

\section{The first decorrelation step}

The above results are quite promising. In order to corroborate these results, we will now compute the actual ambiguity transformation. To show how the ambiguity transformation is constructed, the first step of the transformation will be derived analytically. It follows from (6) that the $L_{2}$ ambiguity is of a better precision than the $L_{1}$ ambiguity. Hence, we will start working on the $L_{1}$ ambiguity. This implies that the first step of the transformation reads as

$$
Z_{1}^{*}=\left[\begin{array}{cc}
1 & -\left[\sigma_{N_{1} N_{2}} \sigma_{N_{2}}^{-2}\right] \\
0 & 1
\end{array}\right],
$$

in which [.] denotes 'rounding to the nearest integer'. From (6) it follows that

$$
\sigma_{N_{1} N_{2}} \sigma_{N_{2}}^{-2}=\frac{\lambda_{2} / \lambda_{1}}{\left(1+2 \sigma_{\Phi}^{2} / \sigma_{P}^{2}\right)} .
$$

If $\sigma_{N_{1} N_{2}} \sigma_{N_{2}}^{-2}$ is less than one half in absolute value, than $\left[\sigma_{N_{1} N_{2}} \sigma_{N_{2}}^{-2}\right]=0$ and transformation (33) reduces to the trivial identity transformation. With $\lambda_{2} / \lambda_{1}=77 / 60$, it follows from (34) however, that

$$
\sigma_{N_{1} N_{2}} \sigma_{N_{2}}^{-2}>1 / 2 \Leftrightarrow \sigma_{\Phi}<\sqrt{(47 / 60)} \sigma_{P} .
$$

This shows, since the precision of the code measurements will never be that precise, that the inequality of (35) will hold true in practice. Hence, transformation (33) will never reduce to the trivial identity transformation. We may therefore conclude that our ambiguity transformation will always result in ambiguities that are less correlated.

In (35), we considered a lower bound for $\sigma_{N_{1} N_{2}} \sigma_{N_{2}}^{-2}$. It is, however, also of interest to consider the upper bound $\sigma_{N_{1} N_{2}} \sigma_{N_{2}}^{-2}<3 / 2$. If namely both this upper bound as well as the lower bound of (35) are satisfied, then $\left[\sigma_{N_{1} N_{2}} \sigma_{N_{2}}^{-2}\right]=1$ and the transformation of (33) becomes

$$
Z_{1}^{*}=\left[\begin{array}{rr}
1 & -1 \\
0 & 1
\end{array}\right],
$$

showing that the first step of our decorrelating ambiguity transformation replaces the $L_{1}$ ambiguity with the widelane ambiguity $N_{w}=N_{1}-N_{2}$. It follows from (34) and $\lambda_{2} / \lambda_{1}$ $=77 / 60$, that $\sigma_{N_{1} N_{2}} \sigma_{N_{2}}^{-2}<3 / 2$ always holds true. Hence, the remarkable conclusion is reached, that the first step of our decorrelating ambiguity transformation will, in practice, always automatically produce the wide-lane ambiguity.

Now that we know that the first decorrelation step will always produce the wide-lane ambiguity, it is also of interest to find out whether a further improvement is generally still possible. Following (36), the second transformationstep is of the form

$$
Z_{2}^{*}=\left[\begin{array}{cc}
1 & 0 \\
-\left[\sigma_{N_{2} N_{\varpi}} \sigma_{N_{w}}^{-2}\right] & 1
\end{array}\right],
$$

with $\sigma_{N_{z} N_{w}}$ being the covariance between the $L_{2}$ ambiguity and the wide-lane ambiguity and with $\sigma_{N_{*}}^{2}$ being the variance of the wide-lane ambiguity. The question is now, under what conditions the following two inequalities are satisfied,

$$
\sigma_{N_{2} N_{w}} \sigma_{N_{w}}^{-2}>-1 / 2 \text { and } \sigma_{N_{2} N_{w}} \sigma_{N_{w}}^{-2}<1 / 2 .
$$

If both inequalities are satisfied, then $\left[\sigma_{N_{N} N_{\nu}} \sigma_{N_{w}}^{-2}\right]=0$, and the conclusion is reached that no further improvement is possible anymore. It follows, using (6) and (36), from $Z_{1}^{*} Q_{N} Z_{1}$, that

$$
\begin{aligned}
\sigma_{N_{2} N_{w}} \sigma_{N_{w}}^{-2}= & \left(\lambda_{1} \lambda_{2}-\lambda_{1}^{2}\left(1+2 \sigma_{\Phi}^{2} / \sigma_{P}^{2}\right)\right) / \\
& \left(\left(\lambda_{2}^{2}+\lambda_{1}^{2}\right)\left(1+2 \sigma_{\Phi}^{2} / \sigma_{P}^{2}\right)-2 \lambda_{1} \lambda_{2}\right) .
\end{aligned}
$$

From this result follows that the first inequality of (38) is always satisfied. The second inequality however, is only satisfied when

$$
\sigma_{\Phi}^{2} / \sigma_{P}^{2}>-1 / 2+2 \lambda_{1} \lambda_{2} /\left(\lambda_{2}^{2}+3 \lambda_{1}^{2}\right)
$$

holds true. Since this implies with $\lambda_{2} / \lambda_{1}=77 / 60$, that $\sigma_{\Phi}$ $>0.228 \sigma_{p}$ must hold true, it follows that the second inequality of (38) will not be satisfied in practice. Hence, the conclusion is reached, that a further improvement in terms of decorrelation is indeed generally possible.

\section{The remaining decorrelation steps}

Since a further improvement is generally still possible after the transformation (36) has been applied, we will now consider the remaining decorrelation steps of the LAMBDA-method. The following two cases for one single epoch $(k=1)$ will be considered: (a) $\sigma_{\Phi}=0.3 \mathrm{~cm}$ and $\sigma_{p}=$ $60 \mathrm{~cm}$; (b) $\sigma_{\Phi}=0.3 \mathrm{~cm}$ and $\sigma_{P}=10 \mathrm{~cm}$. The first decorrelation step, being (36), is the same for both cases. 
In order to quantify the improvement which is obtained through $Z_{1}^{*}$, we compare $Q_{N}$ with $Z_{1}^{*} Q_{N} Z_{1}$. For the first case, this gives

$$
Q_{N}=\left[\begin{array}{ll}
19.884 & 15.493 \\
15.493 & 12.073
\end{array}\right], Z_{1}^{*} Q_{N} Z_{1}=\left[\begin{array}{cc}
0.971 & 3.420 \\
3.324 & 12.073
\end{array}\right]
$$

Although the precision of the first ambiguity has been improved considerably, the improvement in the correlation coefficient is still only marginal. It improved from 0.99995 to 0.99897 . We therefore continue with the second and following steps, until no further decorrelation can be achieved. The resulting decorrelating ambiguity transformation is then obtained as

$$
Z^{*}=\left(\begin{array}{ll}
-7 & 9 \\
-4 & 5
\end{array}\right]=\left(\begin{array}{ll}
1 & 2 \\
0 & 1
\end{array}\right]\left[\begin{array}{cc}
1 & 0 \\
-4 & 1
\end{array}\right]\left[\begin{array}{cc}
1 & -1 \\
0 & 1
\end{array}\right)
$$

Hence, in the present case three steps are required to obtain $Z^{*}$. In order to quantify the improvement which is obtained through $Z^{*}$, we compare the new variancecovariance matrix $Q_{z}=Z^{*} Q_{N} Z$, with the original variancecovariance matrix $Q_{N}$ :

$$
Q_{N}=\left[\begin{array}{ll}
19.884 & 15.493 \\
15.493 & 12.073
\end{array}\right], Q_{z}=\left[\begin{array}{ll}
0.101 & 0.028 \\
0.028 & 0.246
\end{array}\right] \text {. }
$$

This result shows that the correlation coefficient has been improved from $\rho_{N_{1} N_{2}}=0.99995$ to $\rho_{z_{1} z_{2}}=0.179$. Hence, the new ambiguities are much less correlated. Also the elongation of the ambiguity search space has improved. It changed from $e_{N, N_{2}}=206$ to $e_{z_{1} z_{2}}=1.6$. Finally, note the improvement in precision of the ambiguities. The standard deviations have been improved by a factor of about ten: $\sigma_{N_{1}}=4.46$ (cycle), $\sigma_{N_{2}}=3.47$ (cycle) versus $\sigma_{z_{1}}=0.32$ (cycle), $\sigma_{z_{2}}=0.50$ (cycle). This has as consequence that the inequalities of (20), when expressed in terms of the transformed ambiguities, permit a faster search for the integer least-squares solution. The first bound is tighter and the second bound will less likely be a cause for search halting. Based on $\chi^{2}=10$, figure 2 shows both the original and the transformed ambiguity search space. Figure $2 \mathrm{a}$ shows for $k=1$, the very elongated ambiguity search space of the original $L_{1}$ and $L_{2}$ ambiguities. Figure $2 \mathrm{~b}$ shows for $k=1, k=2$ and $k=10$, the transformed ambiguity search space. The drastic improvement is clearly visible. For the three cases shown in figure $2 b$, the numbers of grid points inside the ellipse are respectively 5,3 and 1 . These values may vary slightly when the ambiguity search space is centred at the actual least-squares estimate of the ambiguities.
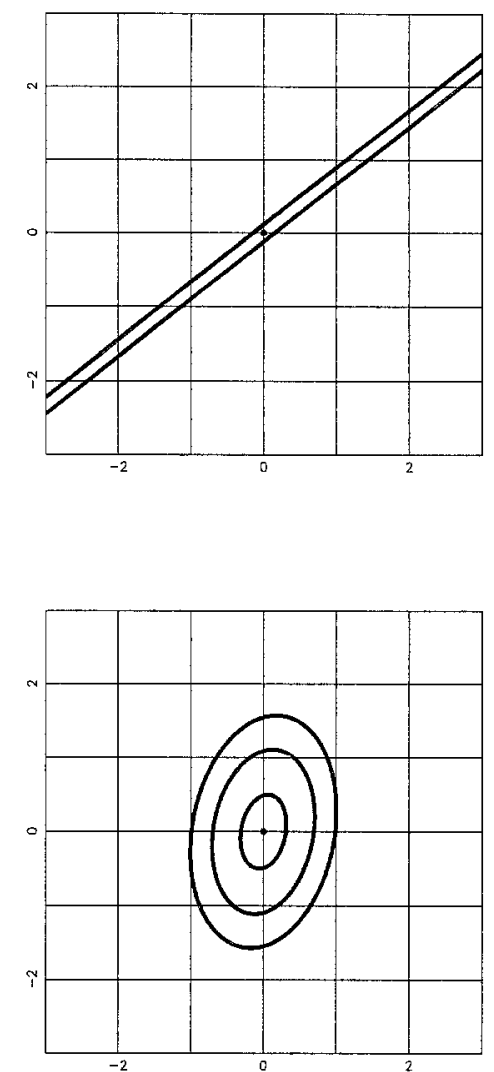

Figure 2: (a) Original ambiguity search space for $Q_{N}$ of (43) with $\chi^{2}=10$ and $k=1$; (b) Transformed ambiguity search space for $Q_{z}$ of (43), with $\chi^{2}=10$ and for $k=1$, $k=2, k=10$.

Let us now consider the second case, being $\sigma_{\Phi}=0.3 \mathrm{~cm}$ and $\sigma_{P}=10 \mathrm{~cm}$. For $Q_{N}$ and $Z_{1}^{*} Q_{N} Z_{1}$ we get

$$
Q_{N}=\left(\begin{array}{ll}
0.553 & 0.430 \\
0.430 & 0.336
\end{array}\right), Z_{1}^{*} Q_{N} Z_{1}=\left[\begin{array}{ll}
0.029 & 0.094 \\
0.094 & 0.336
\end{array}\right] .
$$

Again we see that the precision of the first ambiguity has been improved, but that the improvement in the correlation coefficient is only marginal. It improved from 0.9982 to 0.9645 . We therefore again continue with the second and following steps. As a result we get for $Z^{*}$ :

$$
Z^{*}=\left(\begin{array}{cc}
1 & -1 \\
-3 & 4
\end{array}\right)=\left(\begin{array}{cc}
1 & 0 \\
-3 & 1
\end{array}\right)\left(\begin{array}{cc}
1 & -1 \\
0 & 1
\end{array}\right)
$$


Hence, in this case only two steps are required to obtain $Z^{*}$. In order to quantify the improvement which is obtained through $Z^{*}$, we compare the new variancecovariance matrix $Q_{z}=Z^{*} Q_{N} Z$, with the original variancecovariance matrix $Q_{N}$ :

$$
Q_{N}=\left[\begin{array}{ll}
0.553 & 0.430 \\
0.430 & 0.336
\end{array}\right], Q_{z}=\left[\begin{array}{ll}
0.029 & 0.009 \\
0.009 & 0.026
\end{array}\right]
$$

This result shows that the correlation coefficient has been improved from $\rho_{N_{1} N_{2}}=0.9982$ to $\rho_{z_{1} z_{2}}=0.3243$. Hence, the new ambiguities are much less correlated. Also the elongation of the ambiguity search space has improved. It changed from $e_{N_{1} N_{2}}=34$ to $e_{z_{1} z_{2}}=1.4$. Finally, note the improvement in precision of the ambiguities. The standard deviations have been improved by a factor of about four: $\sigma_{N}$ $=0.74$ (cycle), $\sigma_{N_{2}}=0.58$ (cycle) versus $\sigma_{z_{1}}=0.17$ (cycle), $\sigma_{z_{2}}$ $=0.16$ (cycle). Again based on $\chi^{2}=10$, figure 3 shows both the original and the transformed ambiguity search space. Figure $3 a$ shows for $k=1$, the elongated ambiguity search space of the two original $L_{1}$ and $L_{2}$ ambiguities. Figure $3 \mathrm{~b}$ shows for $k=1, k=2$ and $k=10$, the transformed ambiguity search space. For the three cases shown in figure
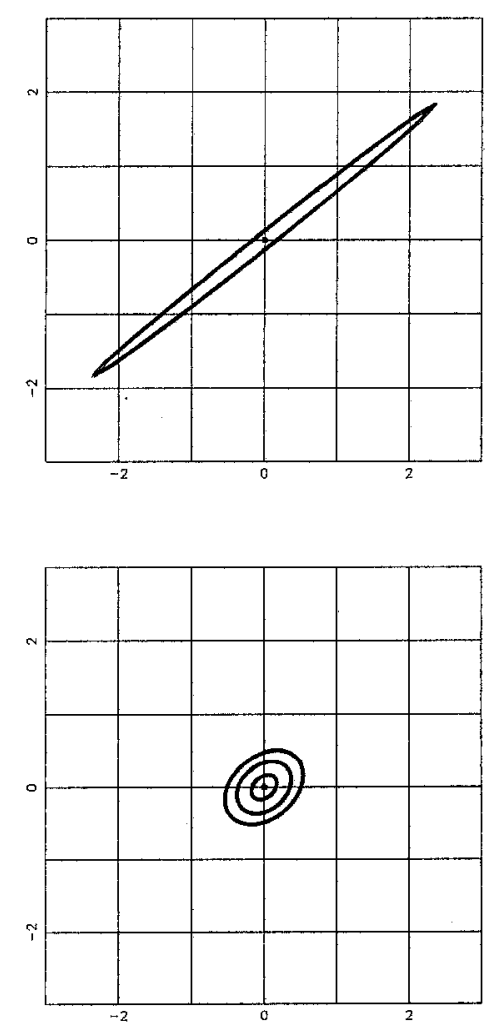

Figure 3: (a) Original ambiguity search space for $Q_{N}$ of (46) with $\chi^{2}=10$ and $k=1$; (b) Transformed ambiguity search space for $Q_{z}$ of (46) with $\chi^{2}=10$ and for $k=1, k=2$, $k=10$.
$3 b$, the numbers of grid points inside the ellipse are all equal to 1 .

\section{Summary}

For the case the relative receiver-satellite geometry is dispensed with, this contribution presented a qualitative analysis of the integer ambiguity estimation problem. The analysis was based on double differenced dual frequency measurements of both carrier phase and code. The ionospheric delays were assumed to be absent. It was emphasized that, in contrast to the single frequency case, a simple rounding of the real-valued $L_{1} / L_{2}$ ambiguity estimates to their nearest integer does not guarantee that one obtains the most likely integer estimates. In order to guarantee this, a search needs to be performed using the ambiguity search space. Based on the analytical form of the variance-covariance matrix of the $L_{1}$ and $L_{2}$ ambiguities of section 2 , the geometry of the ambiguity search space was characterized in section 3 by means of diagnostic measures. These measures were: the elongation (e), the correlation coefficient $(\rho)$, the area of the ambiguity search space $\left(A_{N}\right)$ and the areas of its eigenvalue- and sigma-box $\left(A_{\mu_{N}}, A_{\sigma_{N}}\right)$. In order to have a quick reference, these measures are summarized in table 3 and are given as function of the carrier phase and code measurement precision, $\sigma_{\Phi}$ and $\sigma_{P}$. The expressions in table 3 are suitable approximations to the exact formulae (12), (13), (15), (18) and (32). The approximations are based on the assumption that the variance ratio $\sigma_{\Phi}^{2} / \sigma_{P}^{2}$ is small.

$$
\begin{gathered}
\text { (i) } e_{N_{1} N_{2}} \cong \frac{1}{2}\left(\lambda_{1} / \lambda_{2}+\lambda_{2} / \lambda_{1}\right) \frac{\sigma_{P}}{\sigma_{\Phi}} \quad \text { (iii) } A_{N} \cong \frac{4 \pi \chi^{2}}{\lambda_{1} \lambda_{2} k} \sigma_{P} \sigma_{\Phi} \\
\text { (ii) } \rho_{N_{1} N_{2}} \cong 1-2\left(\frac{\sigma_{\Phi}}{\sigma_{P}}\right)^{2} \\
\text { (v) } A_{\sigma_{s}} \leq \frac{4 \sigma_{\Phi}}{\sqrt{3} \sigma_{P}} A_{\sigma_{N}}
\end{gathered}
$$

Table 3: Diagnostic measures of the ambiguity search space as function of $\sigma_{\Phi}$ and $\sigma_{P}$.

Since $\sigma_{P} / \sigma_{\Phi}$ is large in case of GPS, it directly follows from table 3 , that the ambiguity search space is very elongated ( $e_{N_{1} N_{2}}$ large) and that the $L_{1}$ and $L_{2}$ ambiguities are highly correlated ( $\rho_{N_{1} N_{2}}$ close to 1 ). Elongation and the correlation coefficient react differently however, to an improvement of the code precision. Elongation is to a good approximation linear in $\sigma_{P} / \sigma_{\Phi}$. Hence, by improving the 
code precision, one can force the ambiguity search space to become more sphere-like. The correlation coefficient on the other hand however, is rather insensitive to changes in the precision of the code measurements. Hence, the correlation coefficient of the $L_{1} / L_{2}$ ambiguities will remain close to one for all practical purposes. In fact, the correlation coefficient will only be smaller than one half, when the variance of the code measurements is smaller than twice the variance of the carrier phase measurements.

Since $\sigma_{P} / \sigma_{\Phi}$ is large, table 3 also shows that the area of the enclosing sigma-box, $A_{\sigma_{\mathrm{v}}}$, is very much larger than the area of the ambiguity search space itself. The discrepancy between the two areas can be reduced however, by improving the code precision. It was remarked that the area of the ambiguity search space gives an indication of the number of grid points located in the search space. Table 3 shows that the area $A_{\sigma_{\nu}}$ is governed by the square of $\sigma_{P}$ and that it is independent of $\sigma_{\Phi}$. The area of the ambiguity search space however, depends on $\sigma_{\Phi}$ and is governed by the product $\sigma_{P} \sigma_{\Phi}$. Hence, it is in the area of the ambiguity search space, where the benefit of the very precise carrier phase measurements manifests itself.

The decorrelating ambiguity transformation $Z^{*}$ of the LAMBDA-method was discussed in section 4. This transformation allows one to transform the original $L_{1} / L_{2}$ ambiguities into two new ambiguities that are much less correlated. The correlation coefficient of the transformed ambiguities is always less than or equal to one half in absolute value. Table 3 shows that the transformation results in a drastic decrease in size of the area of the sigma-box, $A_{\sigma,}$ versus $A_{\sigma_{v}}$. Apart from the decrease in the correlation coefficient, the 2D-decorrelating ambiguity transformation also results in a more sphere-like ambiguity search space, having ambiguities with a significantly improved precision. For $\sigma_{\Phi}=0.3 \mathrm{~cm}$, table 4 gives a numerical overview of the precision, elongation and coefficient of both the original and transformed ambiguities, for four different values of $\sigma_{p}$. The results of table 4 are given for $k=1$. They are, however, easily adjusted so as to hold for the case $k>1$. For $k>1$, the standard deviations simply need to be divided by the square-root of $k$. The elongation and the correlation coefficient however, do not change, since they are independent of $k$.

The corresponding ambiguity transformations, $Z^{*}$, are also given in the table. They also hold for the case $k>1$. In section 4 we studied the decorrelation for the limiting case

\begin{tabular}{|c|c|c|c|c|c|c|c|c|c|}
\hline $\begin{array}{c}\sigma_{\Phi}=0.3 \mathrm{~cm} \\
(\mathrm{k}=1)\end{array}$ & $\sigma_{N_{1}}$ & $\sigma_{N_{2}}$ & $e_{N, N}$ & $\mathrm{p}_{N, N_{z}}$ & $Z^{*}$ & $\sigma_{z_{1}}$ & $\sigma_{z_{3}}$ & $e_{z_{j} z_{1}}$ & $\rho_{z_{i z}}$ \\
\hline$\sigma_{P}=60 \mathrm{~cm}$ & 4.46 & 3.47 & 206 & 0.99995 & $\begin{array}{l}-7 \\
-4\end{array}$ & 0.32 & 0.50 & 1.6 & \\
\hline$\sigma_{p}=30 \mathrm{~cm}$ & 2.23 & 1.74 & 103 & 0.9998 & & 0.29 & 0.29 & 1.6 & -0. \\
\hline$\sigma_{P}=10 \mathrm{~cm}$ & 0.74 & 0.58 & 34 & 0.998 & $\begin{array}{l}-3 \\
1-\end{array}$ & 0.16 & 0.17 & 1.4 & 0.3 \\
\hline$\sigma_{P}=3 \mathrm{~cm}$ & 0.23 & 0.18 & 10 & 0.96 & $\begin{array}{r}-2 \\
1 .\end{array}$ & 0.12 & 0.06 & 1.9 & -0.0 \\
\hline
\end{tabular}

Table 4: Precision $(\sigma)$, elongation (e) and correlation $(\rho)$ before and after application of the decorrelating correlation transformation $Z^{*}$.

$\sigma_{\Phi}=0$. The corresponding ambiguity transformation was given as

$$
Z^{*}=\left(\begin{array}{ll}
-60 & 77 \\
-7 & 9
\end{array}\right) \text {. }
$$

This is the optimal transformation for the case the carrier phase measurements are of extreme precision or the code measurements are of very poor precision. In the reversed situation - very precise code measurements and/or carrier phase measurements of poor precision - the optimal ambiguity transformation reduces to the trivial identity transformation. The transformations given in table 4 are therefore the ones that lie between (47) and the identity. In section 5 it was shown how these transformations could be constructed. In section 5 we showed that the first step in constructing $Z^{*}$, always automatically produces the socalled wide-lane ambiguity as a replacement of the $L_{1}$ ambiguity. But, it was also shown that further improvements, which are significant, are generally still possible. Improvements on the wide-lane and $L_{2}$ ambiguity are only impossible, when $\sigma_{\Phi}>0.228 \sigma_{p}$ holds true. One can therefore expect, that one of the two transformed ambiguities will be the wide-lane ambiguity, only when the code measurements are sufficiently precise. This is also evidenced by the results of table 4 . Each of the two last transformations in table 4 transforms such that one of the two transformed ambiguities equals the wide-lane ambiguity. Also observe from table 4 , that neighbouring pairs of the four pairs of transformed ambiguities, have one transformed ambiguity in common.

\section{References}

[1] Blewitt, G. (1989): Carrier phase ambiguity resolution for the Global Positioning System applied to geodetic baselines up to $2000 \mathrm{~km}$. Journal of Geophysical Research, 
Vol. 94, No. B8, pp. 10.187-10.203.

[2] Dedes, G., C. Goad (1994): Real-Time cm-level GPS Positioning of Cutting Blade and Earth Moving Equipment. Proceedings of the 1994 National Technical Meeting ION, San Diego, California, pp. 587-594.

[3] Euler, H.-J., C. Goad (1990): On Optimal Filtering of GPS Dual Frequency Observations without using Orbit Information. Bulletin Geodesique, Vol. 65, pp. 130-143.

[4] Euler, H.-J., H. Landau (1992): Fast GPS Ambiguity Resolution On-The-Fly for Real-Time Applications. Proceedings 6th Int. Geod. Symp. on Satellite Positioning. Columbus, Ohio, 17-20 March 1992, pp. 650-729.

[5] Euler, H.-J., R. Hatch (1994): Comparison of Several AROF Kinematic Techniques. Proceedings ION-94. pp. 363-370.

[6] Frei, E., G. Beutler (1990): Rapid Static Positioning Based on the Fast Ambiguity Resolution Approach FARA: Theory and First Results. Manuscripta Geodaetica, Vol. 15, No. 6, 1990.

[7] Hatch, R. (1982): The Synergism of GPS Code and Carrier Measurements. Proceedings 3th International Geod. Symp. on Satellite Positioning, Las Cruces, New Mexico, 8-12 February, 1982, Vol. 2, pp. 1213- 1231.

[8] Hatch, R. (1986): Dynamic Differential GPS at the Centimeter Level. Proceedings 4th International Geod. Symp. on Satellite Positioning, Austin, Texas, 28 April - 2 May 1986, pp. 1287-1298.

[9] Hatch, R. (1991): Instantaneous Ambiguity Resolution. Proceedings of the IAG International Symposium 107 on Kinematic Systems in Geodesy, Surveying and Remote Sensing, Sept. 10-13, 1990, Springer-Verlag, New York, pp. 299-308.

[10] Teunissen, P.J.G. (1993): Least-Squares Estimation of the Integer GPS Ambiguities. Invited Lecture, Section IV Theory and Methodology, IAG General Meeting, Beijing, China, August 1993. Also in LGR-Series No. 6, 16 p.

[11] Teunissen, P.J.G. (1994a): A New Method for Fast Carrier Phase Ambiguity Estimation. Proceedings IEEE Position Location and Navigation Symposium PLANS'94, pp. 562-573.
[12] Teunissen, P.J.G. (1994b): On the GPS doubledifferenced ambiguities and their partial search spaces. Proceedings Hotine-Marussi Symposium on Mathematical Geodesy, L'Aquila, Italy May 29-June 3, 1994, Geodetic Theory Todcy, Symposium No. 114, IAG, pp. 39-48.

[13] Teunissen, P.J.G., P.J. de Jonge and C.C.J.M. Tiberius (1994): On the Spectrum of the GPS DD-Ambiguities. Proceedings ION GPS-94, Salt Lake City, Utah, USA, September 20-23, 1994, pp. 115-124.

[14] Teunissen, P.J.G., P.J. de Jonge and C.C.J.M. Tiberius (1995): A new way to fix carrier-phase ambiguities. In: GPS-World. April 1995, pp. 58-61.

[15] Teunissen, P.J.G. (1995a): The invertible GPS ambiguity transformations, Manuscripta Geodaetica, September 1995, Vol. 20, No. 6, pp. 489-497.

[16] Teunissen, P.J.G. (1995b): GPS Carrier Phase Ambiguity Fixing Concepts. In: Lecture Notes in Earth Sciences "International School GPS for Geodesy", Chapter 8, 8.1-8.75. To be published by Springer Verlag.

[17] Tiberius, C.C.J.M., P.J. de Jonge (1995a): Fast Positioning using the LAMBDA-Method. In: Proceedings DSNS95, April 24-28, Bergen, Norway.

[18] Tiberius, C.C.J.M., P.J. de Jonge (1995b): Introduction to GPS Surveying (Part 4). In: Geomatics Info Magazine, October 1995, pp. 61-67.

[19] Wübbena, G. (1991): Zur Modellerung von GPS Beobachtungen für die Hochgenaue Positions-bestimmung. Universität Hannover, Germany. 